\title{
Short-Range Order Fluctuation in the Isotropic Phase of Solutions of 4-trans-4'-n-dodecylcyanobiphenyl in Benzene Studied by Nonlinear Dielectric Spectroscopy
}

\author{
P. KȨDZIORA
}

Institute of Molecular Physics, Polish Academy of Sciences

Smoluchowskiego 17, 60-179 Poznań, Poland

(Received September 2, 2004, revised version January 17, 2005;

in final form April 13, 2005)

\begin{abstract}
Nonlinear dielectric spectroscopy is used to study the dynamics of the smectogenic compound 4-trans- $4^{\prime}-n$-dodecylcyanobiphenyl in the isotropic phase as a function of its concentration in benzene. The results obtained reveal that the large, positive amplitude of the field-induced dielectric increment $\left(\Delta \varepsilon \approx 10^{-3}\right)$ and its frequency dependence observed in the nonlinear dielectric effect spectra, even far from the possible isotropic-smectic A type phase transition, are due to short range fluctuations of the electric moment. The nature of molecular formations as a result of the strong intermolecular orientational correlations is discussed.
\end{abstract}

PACS numbers: 64.70.Md, 72.20.Ht, 68.35.Rh

\section{Introduction}

The special interest in the dynamics of calamitic liquid crystals is based on the macroscopic alignment of rod-like molecules caused by the strong anisotropic interactions. This fascinating behavior occurs even in the isotropic phase of liquid crystals in the vicinity of the isotropic to nematic ( $\mathrm{I}-\mathrm{N})$ phase transition. The pretransitional phenomena are due to the existence of a short-range orientational order of the mesogenic molecules leading to the formation of pseudonematic domains in the isotropic phase, which can be observed by various experiments: magnetic [1] and electric [2] birefringence, light scattering [3], optical Kerr ef- 
fect [4-8], and nonlinear dielectric effect [9-13]. As predicted by the Landau-de Gennes theory $[14,15]$, the size of the domains increases as temperature of the liquid approaches to the transition to the nematic phase.

Recently, the direct transition from the isotropic phase to the one of smectic phases has attracted a lot of attention [16-21]. The Kerr effect studies [16], X-ray reflectivity measurements on the free surface of 4-trans-4'-n-dodecylcyanobiphenyl (12CB) [17], the strain birefringence measurements [18], and dielectric studies [19] show that the isotropic-smectic A type (I-SmA) transition is more strong first order than the I-N transition, which is known to be a very weak first order transition. The low-angle X-ray measurements [17] have detected the existence of smectic A type cybotactic groups in the isotropic phase.

In recent papers $[10,11,13]$ we presented the results of the nonlinear dielectric relaxation studies performed for the mesogenic polar compounds 4-trans$-4^{\prime}$-n-hexylcyclohexyl)isothiocyanatobenzene $\left(\mathrm{C}_{6} \mathrm{H}_{13} \mathrm{CyHxPhNCS}, 6 \mathrm{CHBT}\right)$ and 4-trans-4'-n-hexylcyanobiphenyl $\left(\mathrm{C}_{6} \mathrm{H}_{13} \mathrm{PhPhCN}, 6 \mathrm{CB}\right)$ in the vicinity of the isotropic to nematic phase transition. Both in the case of decreasing temperature of the pure mesogenic compound and in the case of increasing its concentration in nonpolar medium at a constant temperature, an approach to the phase transition manifests itself by a critical-like behavior of the nonlinear dielectric strength and the nonlinear relaxation time.

In this paper we present an experimental study of nonlinear dielectric relaxation in the isotropic phase of solutions of the smectogenic 12CB in benzene, which enables us to detect and characterize the pretransitional behavior anticipating the smectic transition. We have shown that the nonlinear dielectric effect (NDE) observed for these solutions is due to the fluctuations of the electric moment.

Applying an additional static electric field of a very high strength to the liquid studied causes the nonlinearity in the dielectric spectroscopy. Weak electric fields tend to align the permanent dipole moments of dilute polar molecules just enough to make the average angle $\theta$ between the moment $\boldsymbol{\mu}$ and the field $\boldsymbol{E}$ slightly different from $\pi / 2$, the average $\langle\cos \theta\rangle$ being usually very small.

At higher field strengths the average $\langle\cos \theta\rangle$ deviates more from zero so that the electric polarization $P$ gradually increases with the field, and finally saturates when $\langle\cos \theta\rangle$ approaches the unity. Long before attaining that regime, however, the onset of saturation may be found from a decrease of the permittivity of the system, which at a particular bias field $E$ quantifies the rate of change of polarization with the measuring field as follows:

$$
\varepsilon_{0}\left(\varepsilon_{E}-1\right)=(\partial P / \partial E)_{E},
$$

wherein $\varepsilon_{0}$ is the permittivity of vacuum and $\varepsilon_{E}$ is the permittivity measured in the presence of a biasing field $E$ of high amplitude.

The role of the high field is not limited to the increasing degree of orientation of dipoles, but it may excite other physicochemical changes contributing to the 
overall polarization, not active at zero field strength. In practice, the observed effect corresponds to the difference of $(\partial P / \partial E)$ terms measured at high $\left(\varepsilon_{E}\right)$ and at zero $(\varepsilon)$ field strength. The effect is best known as the NDE.

\section{Experiment}

4-trans-4'- $n$-dodecylcyanobiphenyl $\left(\mathrm{C}_{12} \mathrm{H}_{25} \mathrm{PhPhCN}, 12 \mathrm{CB}\right)$ was synthesized and purified at the Institute of Chemistry, Military University of Technology, Warsaw. $12 \mathrm{CB}$ has the following sequence of the phases: crystal $-48^{\circ} \mathrm{C}-$ $\mathrm{SmA}-58.5^{\circ} \mathrm{C}$-isotropic. The purity of the compound, checked by chromatography, was $99.5 \%$. Benzene was distilled and stored over the metallic sodium.

The experimental technique used consists of a stationary relaxation method where a low frequency $(85 \mathrm{~Hz})$ electric field with a high amplitude $\left(E_{0}=1.1 \times\right.$ $10^{7} \mathrm{~V} / \mathrm{m}$ ) periodically perturbs the system and the permittivity change,

$$
\Delta \varepsilon=\varepsilon_{E}-\varepsilon,
$$

is being measured by a probing field $E(\omega)$ of high frequency (up to $100 \mathrm{MHz}$ ) with a small amplitude. The details of the method and the set-up used are described in Ref. [22].

\section{Results and discussion}

Figure 1 presents the nonlinear dielectric spectra recorded in the frequency range of $1 \mathrm{MHz}$ to $40 \mathrm{MHz}$ for the solutions of $12 \mathrm{CB}$ in benzene at $25^{\circ} \mathrm{C}$ varying in the concentration from 0.12 to 0.4 mole fraction, i.e. to the limit of the solubility of this mesogen in benzene. The sensitivity of the method is largely determined by the quality factor $Q$ of the resonance that can be set up. As a consequence the
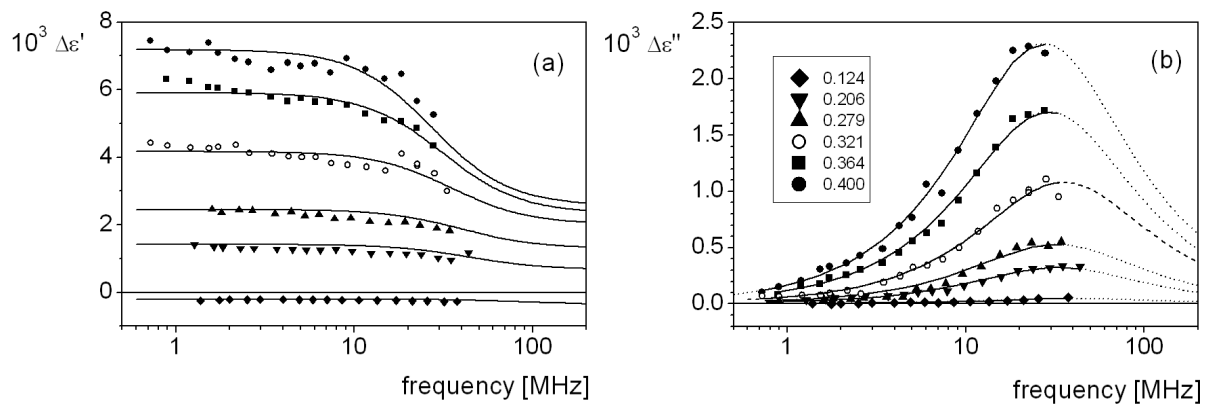

Fig. 1. Frequency dependence of the real (a) and imaginary (b) parts of the nonlinear dielectric increment measured for different molar fractions of $12 \mathrm{CB}$ in benzene, at $25^{\circ} \mathrm{C}, E_{0}=1.1 \times 10^{7} \mathrm{~V} / \mathrm{m}$. The values of the molar fraction of $12 \mathrm{CB}$ are given in the inset. The solid lines correspond to the Debye-type function (9) with a single relaxation time. In the real part the effect of the solvent contribution was subtracted. 
data could only be collected in the frequency domain not too close to the observed Langevin dielectric absorption region, i.e. $\leq 50 \mathrm{MHz}$.

To complete the analysis of the NDE data we have had to make the following assumption: the observed NDE is the result of fluctuations of the electric moment $\boldsymbol{M}$. In the statistical mechanical approach according to Fröhlich [23] one evaluates the overall moment $\boldsymbol{M}$ of a sphere embedded in a continuous dielectric medium with permittivity $\varepsilon$. Clearly, the moment is the vector sum of all molecular dipole moments $\boldsymbol{\mu}$ within the sphere

$$
M=\sum \boldsymbol{\mu}_{i}
$$

The cavity field $\boldsymbol{G}$ that is felt by the particles in the sphere is given as

$$
\boldsymbol{G}=\left(\frac{3 \varepsilon}{2 \varepsilon+1}\right) \boldsymbol{E}
$$

The average moment is calculated as the projection of $\boldsymbol{M}$ on the field direction defined by the unit vector $\boldsymbol{e}$ as follows

$$
\langle\boldsymbol{M} \cdot \boldsymbol{e}\rangle=\frac{\int \mathrm{d} Z(\boldsymbol{M} \cdot \boldsymbol{e}) \exp ((\boldsymbol{M} \cdot \boldsymbol{G}) / k T)}{\int \mathrm{d} Z \exp ((\boldsymbol{M} \cdot \boldsymbol{G}) / k T)},
$$

where $Z$ represents all the spatial and orientational coordinates of the particles. The moment can now be written as a Taylor expansion in terms of the cavity field

$$
\langle\boldsymbol{M} \cdot \boldsymbol{e}\rangle=\left(\frac{\partial\langle\boldsymbol{M} \cdot \boldsymbol{e}\rangle}{\partial G}\right)_{0} G+\left(\frac{\partial^{3}\langle\boldsymbol{M} \cdot \boldsymbol{e}\rangle}{\partial G^{3}}\right)_{0} \frac{G^{3}}{3 !}+\ldots
$$

where even terms have disappeared due to the symmetry. This expression may be written more explicitly as

$$
\langle\boldsymbol{M} \cdot \boldsymbol{e}\rangle=\frac{\left\langle M^{2}\right\rangle_{0}}{3 k T} G+\left(3\left\langle M^{4}\right\rangle_{0}-5\left\langle M^{2}\right\rangle_{0}^{2}\right) \frac{G^{3}}{90(k T)^{3}}
$$

with $\left\langle(\boldsymbol{M} \cdot \boldsymbol{e})^{n}\right\rangle_{0}=\left\langle M^{n} \cos ^{n} \theta\right\rangle_{0}=\left\langle M^{2}\right\rangle_{0} /(n+1)$ for even $n$. Note the subscript 0 indicates the absence of the field. The first term is recognized as the usual contribution to the low-field polarization, while the second term corresponds to the NDE. Indeed, the permittivity is to be understood as

$$
\varepsilon_{0} \varepsilon_{E}=\frac{1}{V} \frac{\partial\langle\boldsymbol{M} \cdot \boldsymbol{e}\rangle_{E}}{\partial E},
$$

where $V$ is the volume of the sphere. The term $\left(3\left\langle M^{4}\right\rangle_{0}-5\left\langle M^{2}\right\rangle_{0}^{2}\right)$, which corresponds to the NDE, we can split up into two parts: $5\left(\left\langle M^{4}\right\rangle_{0}-\left\langle M^{2}\right\rangle_{0}^{2}\right)$, which represents the fluctuation of $M^{2}$ (positive contribution), and a term of the orientational Langevin saturation $-2\left\langle M^{4}\right\rangle_{0}$ (negative contribution). To this last term we have assigned the orientation relaxation function derived for isolated dipolar molecules, whereas for the fluctuational part a simple Debye relaxation function $1 /(1+\mathrm{i} \omega \tau)$ was taken into account. 
From the data in Fig. 1 it is obvious that a growing positive contribution to the NDE is being generated with increasing concentration of the mesogenic compound, i.e. the importance of the fluctuations of the electric moment $M$ increases with increasing concentration.

Three features of the spectra presented in Fig. 1 seem to be important. First of all, the field-induced dielectric increments $(\Delta \varepsilon)$ are positive and the values of the increment are of the order of $10^{-3}$, i.e. the same order like that measured for nematogenic solutions of $6 \mathrm{CB}$ and $6 \mathrm{CHBT}$ in benzene. Secondly, the frequency dependence of the increment can be perfectly described by a simple Debye-type function with a single relaxation time $(\tau)$

$$
\Delta \varepsilon=\Delta \varepsilon^{\prime}-\mathrm{i} \Delta \varepsilon^{\prime \prime}=\frac{\Delta \varepsilon^{0}}{1+\mathrm{i} \omega \tau}
$$

where $\Delta \varepsilon^{\prime}$ and $\Delta \varepsilon^{\prime \prime}$ denote, respectively, the real and imaginary parts of the nonlinear dielectric increment, $\Delta \varepsilon^{0}$ is the strength of the nonlinear effect, and $\omega$ is the angular frequency of the probing field $E(\omega)$.

The values of the relaxation time resulting from the best fitting of Eq. (9) to the experimental nonlinear dielectric spectra are depicted in Fig. 2 as a function of concentration. The strength and the relaxation time for the $12 \mathrm{CB}$ solutions were compared with such values of solutions of mesogenic $6 \mathrm{CB}$ studied previously [13]. And thirdly, the data presented in Fig. 2 show that both the strength of the nonlinear dielectric increment and the nonlinear relaxation time depend on the concentration of the $12 \mathrm{CB}$ solutions and grow with an increase in concentration. It was shown that the nonlinear relaxation observed in $6 \mathrm{CB}$ solutions is due to the randomization of the pseudonematic domains. P.G. de Gennes extended the Landau theory of the second order phase transitions to the weak first order
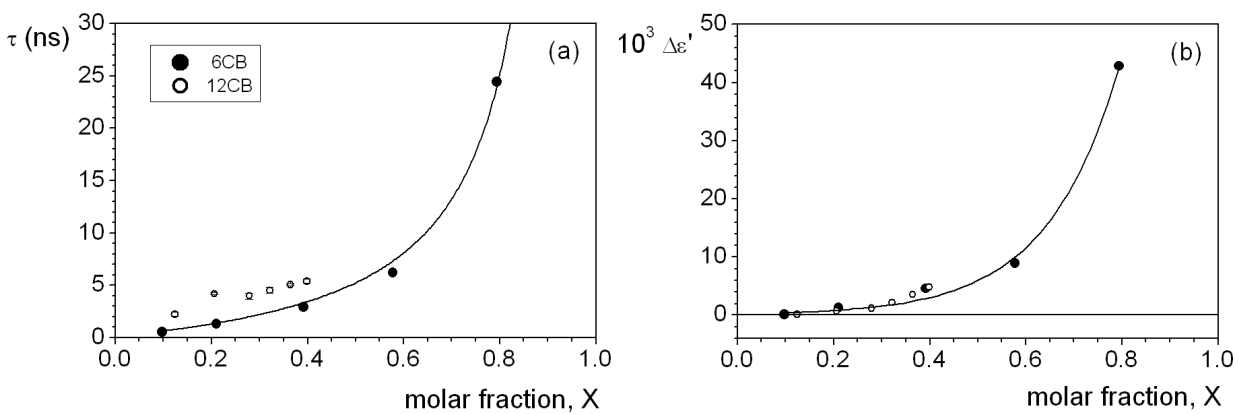

Fig. 2. Concentration dependence of the relaxation time (a) and the strength of the nonlinear dielectric increment (b) for solutions of 6CB (filled circles) [13] and 12CB (open circles) in benzene at $25^{\circ} \mathrm{C}$. The sizes of the circles are about the order of uncertainty for relaxation time and strength of nonlinear dielectric increment. The solid line through the points of the relaxation time is the Landau-de Gennes theoretical curve for $6 \mathrm{CB}$ solutions. 
nematic-isotropic phase transition $[14,15]$ to analyze the slow time scale and long distance scale dynamics of pseudonematic domains. The Landau-de Gennes (LdG) theory was used for interpretation of the pretransitional phenomena observed in the isotropic mesogenic liquids in the vicinity of the nematic phase obtained with the light scattering [3, 24] and the optical Kerr effect [4-8] methods. The LdG theory also correctly describes the temperature and concentration dependences of the NDE relaxation in the pretransitional region of pure 6CHBT [10], as well as 6CHBT and $6 \mathrm{CB}$ solutions $[11,13]$.

On the contrary to the $12 \mathrm{CB}$ solutions, the nonlinear dielectric spectra of solutions of nonmesogenic carboxylic acid (MCHA) [12] point out for no well-organized molecular system, which in the strong electric field relaxes with no well-defined relaxation time. A great variety of intermolecular entities formed make the spectrum very broad. The lack of similarity of nonlinear dielectric behaviors of solutions of both studied compounds, 12CB and MCHA, leads to the conclusion that $12 \mathrm{CB}$ solutions in benzene do not behave like solutions of nonmesogenic compounds.

The magnitude of the positive nonlinear dielectric increment and concentration dependence of nonlinear relaxation time, suggest the formation of the microdomains with a large dipole moment in isotropic phase of $12 \mathrm{CB}$ 's solutions in benzene. A strong electric field can shift the equilibrium between isotropic phase and mesogenic domains toward the polar domains. The nonlinear dielectric effect due to this process is large $\left(\Delta \varepsilon \approx 10^{-3}\right)$ because the short-ranged ordering in the microdomains leads to the strong polarity.

In order to check whether such domains exist in the solutions studied, firstly we examine whether the nonlinear relaxation observed in $12 \mathrm{CB}$ solutions is due to the randomization of the domain, analogous to the pseudonematic domains.

In nematogens, the pretransitional phenomena are due to the pretransitional fluctuation leading to the formation of the pseudonematic domains in the isotropic liquid. The correlation length of the domains increases as the temperature of pure liquid $[7,8,10,25-27]$ or as the concentration of mesogenic molecules in solutions approaches the transition to the nematic phase [11-13], and such phenomena can be quantitatively described in terms of the LdG theory. It was shown [11] that the nonlinear relaxation time $\tau$ measured as a function of concentration of the mesogenic compound fulfills the following equation:

$$
\tau(X)=\frac{V_{\mathrm{eff}}^{*} \eta(X)}{k T\left(X^{*}-X\right)^{\alpha}}
$$

analogous to that resulting from the LdG theory for dependence of the relaxation time on temperature [15]. In Eq. (10) $X$ denotes the molar fraction of the mesogenic solute, $\eta$ - the viscosity of the solution, $V_{\mathrm{eff}}^{*}$ - the effective volume of the relaxing entity and $k$ - the Boltzmann constant. $X^{*}$ stands for the concentration of virtual transition of the second order, in analogy to $T^{*}$ in the LdG theory, and 
$\alpha$ is the critical exponent. Equation (10) can be used for determination of the concentration $X^{*}$ of the virtual transition of the second order, provided that the experimental dependence $\eta / \tau$ on molar fraction $X$ is linear and the slope of the line is negative.

Figure 3 shows that the dependence of $\eta / \tau$ vs. molar fraction for $12 \mathrm{CB}$ solutions is quite different from that for $6 \mathrm{CB}$ solutions. The limited solubility of $12 \mathrm{CB}$ in benzene makes the comparison of the both dependences of $\eta / \tau$ on molar fraction in the same concentration's region impossible. However, as seen in Fig. 3, the linear dependence $\eta / \tau$ on molar fraction $X$ is quite good linear (with a negative slope) also in the concentration region below 0.45 molar fraction for $6 \mathrm{CB}$ 's solutions, i.e. in the attainable region of concentration for $12 \mathrm{CB}$ solutions. The dependence of $\eta / \tau$ on molar fraction of $12 \mathrm{CB}$ in benzene is, within the experimental errors, concentration independent and the concentration $X^{*}$ cannot be determined. In consequence, according to our expectation for the direct isotropic to smectic A phase transition, the LdG theory cannot be used to the explanation of these experimental data. Such a transition is strong first order, without the virtual transition of the second order at the temperature $T^{*}$ or concentration $X^{*}$. It means that the nonlinear dielectric relaxation observed in $12 \mathrm{CB}$ solutions is not due to the randomization of the domains similar to the pseudonematic domains existing in the isotropic solutions in the vicinity of the I-N phase transition.

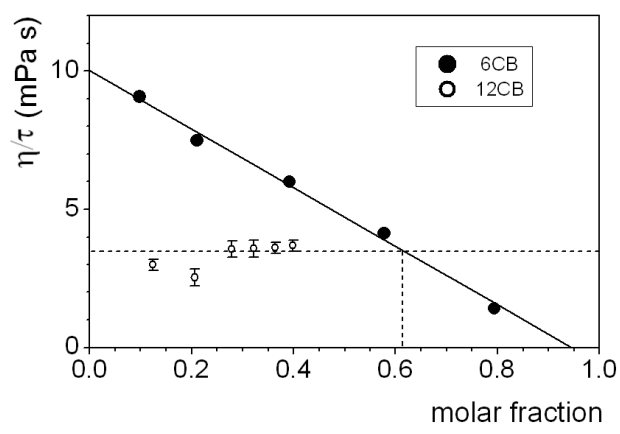

Fig. 3. Concentration dependence of $\eta / \tau$ of $6 \mathrm{CB}$ (filled circles) [13] and 12CB (open circles) solutions in benzene. The straight line is a linear fit through the $6 \mathrm{CB}$ data.

Recently, Bellini et al. [20, 21] pointed out that for the 10CB the direct I-SmA phase transition takes place through the nucleation of smectic domains. They found that the size of the smectic domains is at least $220 \AA$ and the correlation length remains almost constant in the whole two-phase coexisting region. This behavior contrasts with that of materials where the smectic phase develops from a locally orientationally ordered nematic $\lambda$ phase and can be interpreted as a nucleation-type process. The constant values of $\eta / \tau$ ratio (Fig. 3) imply that the correlation length $\xi$ of possible domains does not depend on a distance from the I-SmA phase transition (in the concentration scale) (Fig. 4). This behavior, 


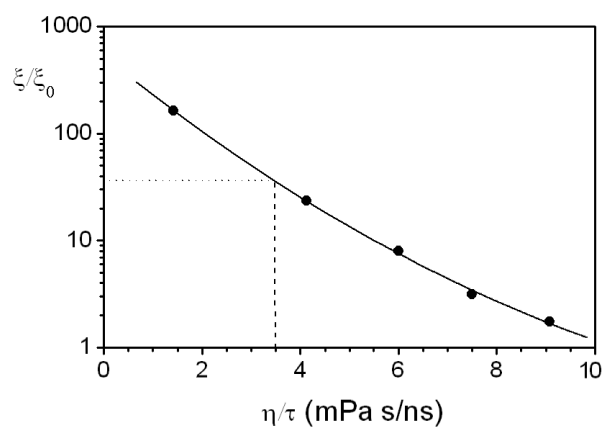

Fig. 4. The dependence of the correlation length $\xi$ on the ratio $\eta / \tau$ for the solutions of $6 \mathrm{CB}$ in benzene. The correlation length $\xi$ was determined from relation $\xi(N)=$ $\xi_{0}\left(\frac{N^{*}}{N^{*}-N}\right)^{2}[11]$ and Eq. (10). The molecular dimension $\xi_{0}$ of $6 \mathrm{CB}$ molecule is equal to about $8 \AA$.

combined with the above-mentioned observations for 10CB [20, 21], could suggest that the dynamic nonlinear dielectric properties measured in solutions of $12 \mathrm{CB}$ are probably due to the nucleation of smectic domains in isotropic solutions. However, a large distance from a possible I-SmA phase transition, in which the studies were performed, hinders us from drawing conclusions on the presence of such domains in isotropic solutions of smectogenic $12 \mathrm{CB}$ in benzene.

\section{Conclusions}

Until now the NDE studies of mesogenic compounds dissolved in a nonpolar medium was limited to the systems with I-N phase transitions [11-13]. In this paper the nonlinear dielectric spectroscopy has been used to study the dynamics of the mesogenic 4-trans-4'-n-dodecylcyanobiphenyl (12CB) in the isotropic phase as a function of concentration in benzene. We have been able to show that the nonlinear dielectric effect for solutions of mesogens is due to fluctuations of the electric moment. Some features of the spectra such as the large positive amplitude of the field-induced dielectric increment $\left(\Delta \varepsilon \approx 10^{-3}\right)$, the frequency dependence of the increment can be perfectly described by a simple Debye-type function with a single relaxation time $(\tau)$ and its concentration dependences, suggest that in these solutions there are the strong intermolecular orientational correlations. The most remarkable fact is that these features follow the behavior expected for pretransitional fluctuations of the electric moment in the vicinity of the isotropic to liquid crystalline phase transition. As we have shown in previously papers [10-14] these fluctuations lead to the formation of the domains with short-ranged ordering in the isotropic phase. The size of these domains increases as the temperature or concentration of mesogenic molecules approaches the transition to the nematic phase. However, the fact that the experimental results of the nonlinear dielectric relaxation cannot be explained in the frame of the LdG theory allows us to exclude 
the possibility of the formation of such domains in $12 \mathrm{CB}$ solutions. In view of a poor solubility of $12 \mathrm{CB}$ in benzene, the measurements were undertaken far from the possible I-SmA phase transition (in the concentration scale). This hinders us from drawing firm conclusions on the nature of molecular formations as a result of the strong intermolecular orientational correlations.

\section{Acknowledgments}

This work was supported by the Fonds voor Wetenschappelijk Onderzoek Vlaanderen, Belgium, in the framework of the agreement for scientific cooperation with the Polish Academy of Sciences.

\section{References}

[1] J.D. Litster, T.W. Stinson III, J. Appl. Phys. 41, 996 (1970).

[2] J.C. Filippini, Y. Poggi, Phys. Lett. A 65, 30 (1978).

[3] T.D. Gierke, W.H. Flygare, J. Chem. Phys. 61, 2231 (1974).

[4] G.K.L. Wong, Y.R. Shen, Phys. Rev. Lett. 30, 895 (1973).

[5] F.W. Deeg, M.D. Fayer, J. Chem. Phys. 91, 2269 (1989).

[6] F.W. Deeg, S.R. Greenfield, J.J. Stankus, V.J. Newell, M.D. Fayer, J. Chem. Phys. 93, 3503 (1990).

[7] J.J. Stankus, R. Torre, C.D. Marshall, S.R. Greenfield, A. Sengupta, A. Tokmakoff, M.D. Fayer, Chem. Phys. 194, 213 (1992).

[8] J.J. Stankus, R. Torre, M.D. Fayer, J. Phys. 97, 9478 (1993).

[9] A. Drozd-Rzoska, S.J. Rzoska, J. Zioło, Phys. Rev. E 54, 6452 (1996).

[10] P. Kȩdziora, J. Jadżyn, L. Hellemans, Phys. Rev. E 66, 031702 (2002).

[11] P. Kȩdziora, J. Jadżyn, L. Hellemans, Phys. Rev. E 66, 021709 (2002).

[12] P. Kȩdziora, J. Jadżyn, L. Hellemans, J. Phys. Chem. A 107, 5650 (2003).

[13] P. Kędziora, Acta Phys. Pol. A 104, 45 (2003).

[14] P.G. de Gennes, Mol. Cryst. Liq. Cryst. 12, 193 (1971).

[15] P.G. de Gennes, Physics of Liquid Crystals, Oxford University Press, Oxford 1974.

[16] H.J. Coles, C. Strazielle, Mol. Cryst. Liq. Cryst. Lett. 49, 259 (1979).

[17] B.M. Ocko, A. Braslau, P.S. Peshau, J. Als-Nielsen, M. Deutsch, Phys. Rev. Lett. 57, 94 (1986).

[18] M. Olbrich, H.R. Brand, H. Finkelmann, K. Kawasaki, Europhys. Lett. 31, 281 (1995).

[19] A. Drozd-Rzoska, S.J. Rzoska, J. Zioło, Phys. Rev. E 61, 5349 (2000).

[20] T. Bellini, A.G. Rappaport, N.A. Clark, B.N. Thomas, Phys. Rev. Lett. 77, 2507 (1996).

[21] T. Bellini, N.A. Clark, D.R. Link, J. Phys., Condens. Matter 15, S175 (2003).

[22] L. Hellemans, L. De Maeyer, J. Chem. Phys. 63, 3490 (1975). 
[23] H. Fröhlich, Theory of Dielectrics, 2nd ed., Oxford University Press, London 1958.

[24] T.W. Stinson III, J.D. Litster, Phys. Rev. Lett. 25, 503 (1970).

[25] S.D. Gottke, D.D. Brace, H. Cang, B. Bagchi, M.D. Fayer, J. Chem. Phys. 116, 360 (2002).

[26] S.D. Gottke, H. Cang, B. Bagchi, M.D. Fayer, J. Chem. Phys. 116, 6339 (2002).

[27] H. Cang, J. Li, M.D. Fayer, Chem. Phys. Lett. 366, 82 (2002). 